\title{
Mapping of mechanical strain induced by thin and narrow dielectric stripes on InP surfaces
}

\author{
Jean-Pierre Landesman, ${ }^{1,2, *}$ Daniel T. Cassidy, ${ }^{2}$ Marc Fouchier, ${ }^{3}$ Christophe Levallois, ${ }^{4}$ \\ Erwine Pargon, ${ }^{3}$ Névine Rochat, ${ }^{5}$ Merwan Mokhtari, ${ }^{1}$ Juan Jiménez, ${ }^{6}$ and Alfredo Torres ${ }^{6}$ \\ ${ }^{1}$ Université de Rennes 1, CNRS, IPR (Institut de Physique de Rennes)-UMR 6251, F-35000 Rennes, France \\ ${ }^{2}$ McMaster University, Department of Engineering Physics, 1280 Main Street West, Hamilton, Ontario L8S 4L7, Canada \\ ${ }^{3}$ Université Grenoble Alpes, CNRS, LTM (Laboratoire des Technologies de la Microélectronique), F-38000 Grenoble, France \\ ${ }^{4}$ Univ Rennes, INSA Rennes, CNRS, Institut FOTON-UMR 6082, F-35000 Rennes, France \\ ${ }^{5}$ CEA, LETI, Minatec Campus, F-38054 Grenoble, France \\ ${ }^{6}$ GdS Optronlab, Dpto. Fisica de la Materia Condensada, Universidad de Valladolid, 47011 Valladolid, Spain \\ *Corresponding author: Jean-Pierre.Landesman@univ-rennes1.fr
}

Received 1 May 2018; revised 24 June 2018; accepted 25 June 2018; posted 26 June 2018 (Doc. ID 330483); published 17 July 2018

\begin{abstract}
We investigated deformation of InP that was introduced by thin, narrow, dielectric $\operatorname{SiN}_{\mathrm{x}}$ stripes on the (100) surface of InP substrates. Quantitative optical measurements were performed using two different techniques based on luminescence from the InP: first, by degree of polarization of photoluminescence; and second, by cathodoluminescence spectroscopy. The two techniques provide complementary information on deformation of the InP and thus together provide a means to evaluate approaches to simulation of the deformation owing to dielectric stripes. Ultimately, these deformations can be used to estimate changes in refractive index and gain that are a result of the stripes. (c) 2018 Optical Society of America
\end{abstract}

OCIS codes: (160.6000) Semiconductor materials; (250.5230) Photoluminescence; (250.1500) Cathodoluminescence; (310.4925) Other properties (stress, chemical, etc.); (310.5448) Polarization, other optical properties; (240.0310) Thin films.

https://doi.org/10.1364/OL.43.003505

Stress and strain are important issues, generally speaking, for processing of photonic devices. Stress and strain affect operating characteristics of photonic devices through strain-induced changes in refractive index and in optical gain, and through dependence of reliability on strain.

Optical indices can be modified, either unintentionally or on purpose, to create guiding structures through local strain control.

Several groups have worked in the past on demonstration of feasibility of photo-elastic waveguides in semiconductor materials, where the guiding structure is defined through application of mechanical stress via a patterned dielectric film at the surface [1-5].

The ability to measure deformations accurately, with requisite spatial resolution and sensitivity to all elements of the stress and strain tensors, would be of value in the development of photoelastic waveguides, in device development, and in reliability programs. This ability would be a step towards accurate feedback that could be used to optimize the fabrication of photo-elastic waveguides in particular, and photonic devices in general.

Our purpose in this Letter is to demonstrate an innovative approach to this problem by the combined use of two optical techniques based on exploitation of the luminescence from strained III-V semiconductor materials and by comparison to simulations of the strain induced by the $\mathrm{SiN}_{\mathrm{x}}$ films. Measurements were made on (100) InP substrates that had $500 \mathrm{~nm}$ thick $\mathrm{SiN}_{\mathrm{x}}$ films with widths of $6 \mu \mathrm{m}, 10 \mu \mathrm{m}$, and $20 \mu \mathrm{m}$ and lengths of $3 \mathrm{~mm}$ deposited on them. We also use this opportunity to compare and contrast results obtained by the two optical techniques.

The thermal strain difference between an InP substrate and $\mathrm{Si}_{3} \mathrm{~N}_{4}$ is estimated to be $0.4 \times 10^{-3}$ using coefficients of thermal expansion (CTE) of $\alpha_{\mathrm{InP}}=4.6 \times 10^{-6} \mathrm{~K}^{-1}$ and $\alpha_{\mathrm{Si}_{3} \mathrm{~N}_{4}}=3.3 \times 10^{-6} \mathrm{~K}^{-1}$, and a temperature difference of $\Delta T=$ $(300-20)^{\circ} \mathrm{C}$. These parameters give roughly the correct magnitude but the wrong sign for predicted strain as compared to our measurements. These simple facts illustrate the need for accurate measurements of strain; strain cannot necessarily be estimated directly from differences in CTE and temperature. A $\mathrm{SiN}_{\mathrm{x}}$ film on $\mathrm{InP}$ appears to have an intrinsic, anisotropic strain, similar to what was found by Tien and $\mathrm{Lin}$ for $\mathrm{SiN}_{\mathrm{x}}$ on Si or glass [6]. Tien and Lin [6] deduced a CTE for their $\mathrm{SiN}_{\mathrm{x}}$ films of $3.27 \times 10^{-6} \mathrm{~K}^{-1}$ regardless of the substrate, but found that intrinsic strain in $\mathrm{SiN}_{\mathrm{x}}$ was different for glass and $\mathrm{Si}$ substrates, which further demonstrates need for accurate measurement of strain.

We use two different optical techniques that provide complementary information on the deformations induced by $\mathrm{SiN}_{\mathrm{x}}$ films on (100) InP substrates. Measurement of the degree of polarization (DOP) of photoluminescence (PL) yields information on the difference of in-plane deformations, i.e., non-biaxial deformation, and probes close to the surface, on the order of 
$100 \mathrm{~nm}$ for excitation at $633 \mathrm{~nm}$, with a spatial resolution that is limited to $\gtrsim 1 \mu \mathrm{m}$. Spectral shift cathodoluminescence (CL) yields information on a hydrostatic-equivalent deformation with a spatial resolution that is much better than $1 \mu \mathrm{m}$ and probes a volume that depends on the accelerating voltage. For an accelerating voltage of $10 \mathrm{kV}$, the depth below the surface for maximum electron-hole (eh) pair generation is $\approx 200 \mathrm{~nm}$, but generation of eh pairs is non-negligible up to $\approx 800 \mathrm{~nm}$ [7].

The CL and DOP data show similar patterns for measurements on cleaved (011) facets and on (100) surfaces. The difference of the deformation dependence of luminescence for CL and DOP provides feedback for the selection of accurate approaches to simulation.

The data were fit to an edge-force model [8], which seems to account well for salient features in the (100) data, but does not include anisotropy in the deformation induced by the $\mathrm{SiN}_{\mathrm{x}}$ films, which is observed from DOP measurements. Straightforward 2D and 3D finite element method (FEM) simulations were also performed to understand the CL and DOP measurements. None of the simulations fit significantly better than any given simulation for either surface, suggesting that the situation is significantly more complicated than a uniformly deformed film on a (100) InP substrate.

$500 \mathrm{~nm}$ thick $\mathrm{SiN}_{\mathrm{x}}$ films were deposited on $50 \mathrm{~mm} \operatorname{InP}(100)$ wafers by a plasma enhanced chemical vapor deposition technique using $\mathrm{SiH}_{4} / \mathrm{NH}_{3} / \mathrm{N}_{2}$ precursors at $300^{\circ} \mathrm{C}$ and 2.5 Torr. E-beam lithography was used to define photoresist patterns on the $\mathrm{SiN}_{\mathrm{x}} / \mathrm{InP}$ substrates. Plasma etching was carried out in an inductively coupled plasma reactor. The photoresist patterns were transferred into the $\mathrm{SiN}_{\mathrm{x}}$ layer using a $\mathrm{CF}_{4} / \mathrm{CH}_{2} \mathrm{~F}_{2} / \mathrm{Ar}$ plasma with an etch rate of $\approx 150 \mathrm{~nm} / \mathrm{min}$. The photoresist mask was stripped with an $\mathrm{O}_{2}$ plasma.

Figure 1 is a schematic diagram of a $\operatorname{SiN}_{x}$ film on an InP substrate. The samples were cleaved perpendicular to the $y$ axis of the stripes. Measurements were made on the top surfaces $[x-y$ or (100) planes] and on cleaved $[x-z$ or (011) planes] surfaces.

It has been demonstrated that the DOP of PL from InP or GaAs is proportional to the difference of the strains in the material in the plane of the measured surface [9]. For measurements on the top surface,

$$
D O P_{z}=\frac{L_{x}-L_{y}}{L_{x}+L_{y}}=-\left|K_{z}\right| \times\left(\epsilon_{x x}-\epsilon_{y y}\right),
$$

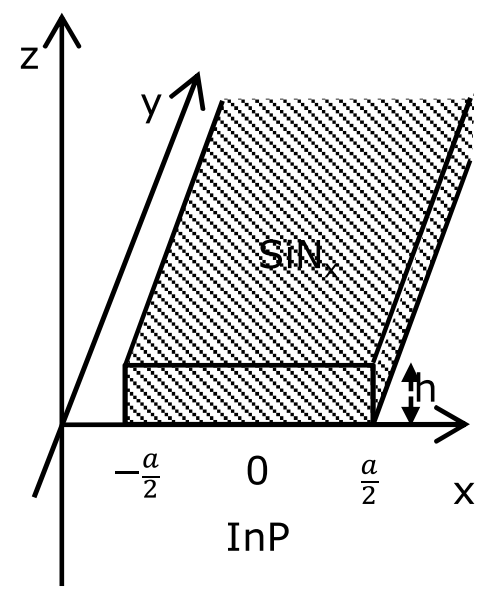

Fig. 1. Geometry of a $\operatorname{SiN}_{\mathrm{x}}$ stripe on an $\operatorname{InP}(100)$ surface. and for measurements on a cleaved facet,

$$
\mathrm{DOP}_{y}=\frac{L_{x}-L_{z}}{L_{x}+L_{z}}=-\left|K_{y}\right| \times\left(\epsilon_{x x}-\epsilon_{z z}\right) \text {. }
$$

Note that the subscript on DOP gives the direction of the normal of the surface under measurement and that strain is $>0$ for tension. For InP, $\left|K_{y}\right|=65 \pm 10$ [9]. $L_{i}$ is the measured intensity of the PL for light polarized along an $i$ direction, $i=x$, $y$, or $z . \epsilon_{x x}, \epsilon_{y y}$, and $\epsilon_{z z}$ are the normal (or tensile) components of the strain tensor.

Measurements of the DOP provide information on the difference of the normal components of strain with noise levels equivalent to strains of $\lesssim 10^{-5}$ and a spatial resolution in the 1-2 $\mu \mathrm{m}$ range.

The CL spectra were acquired at room temperature using a hyperspectral mapping CL scanning electron microscope Rosa 4634. An electron beam acceleration voltage of $10 \mathrm{kV}$ was used. We assume that the peak shift of the band-to-band luminescence relative to a stress-free reference, $\Delta E_{\text {peak }}$, is related to the hydrostatic stress,

$$
\Delta E_{\text {peak }}=k \times\left(\sigma_{x x}+\sigma_{y y}+\sigma_{z z}\right),
$$

with $k=-11 \times 10^{-8} \mathrm{meV} / \mathrm{Pa}$ for InP [10]. $\sigma_{x x}, \sigma_{y y}$, and $\sigma_{z z}$ are the normal (or tensile) components of the stress tensor. The peak shift of CL should be invariant to the direction of observation, unlike DOP, since $\Delta E_{\text {peak }}$ is proportional to the trace of a tensor. However, similar to DOP, we use a subscript on CL to indicate the normal of the surface that is being measured. Figure 2 shows spectrally resolved $\mathrm{CL}_{100}$ signals from a region under compression and from a region from an unstressed reference substrate. The figure shows the peak shift owing to compression and the quality of the data. The peak shift was obtained by careful determinations of the energies of the peak maxima for an unstressed reference and samples through fitting the regions in the vicinities of the peaks of the spectrally resolved luminescences with Gaussians. The accuracy of this procedure appears to be on the order of $0.01 \mathrm{meV}$.

Note that the DOP of PL and CL techniques have different spatial resolutions; roughly, DOP would be limited to stripes broader than $6 \mu \mathrm{m}$, whereas the higher spatial resolution of the $\mathrm{CL}$ permits measuring narrower stripes. Note also that the CL peak shifts are proportional to the sum of the normal stresses, whereas the DOP is proportional to a difference. Thus, DOP should be blind to the local average strain, whereas CL peakshift data see fully the local average stress but might be insensitive to differences between normal stresses, particularly if the

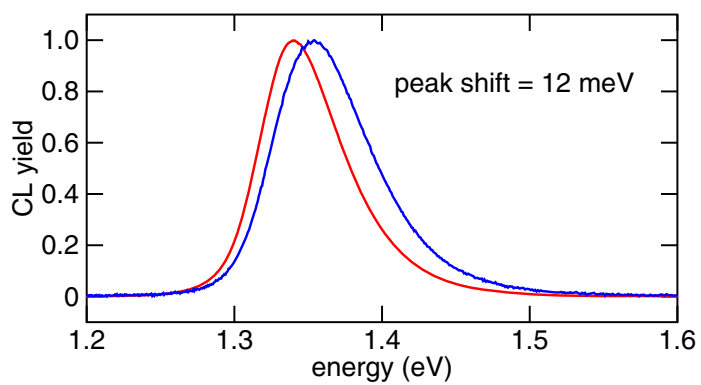

Fig. 2. $\mathrm{CL}_{100}$ spectra from a reference wafer (red) and from a region with compressive stress (blue). 


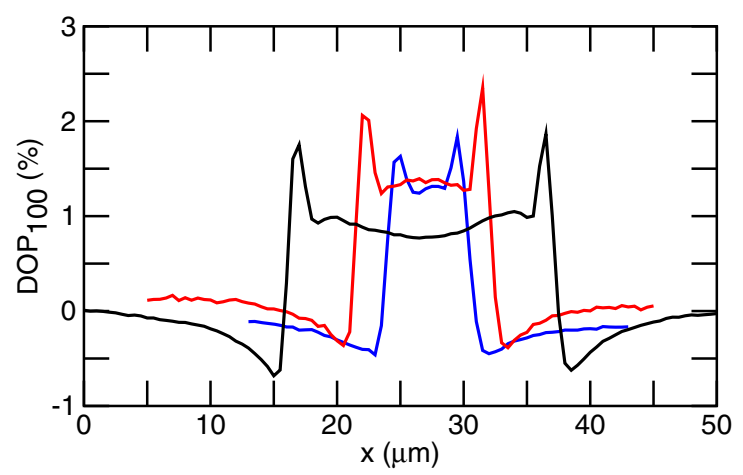

Fig. 3. $\mathrm{DOP}_{100}$ curves measured for $\mathrm{SiN}_{\mathrm{x}}$ stripes with widths of $20 \mu \mathrm{m}$ (black), $10 \mu \mathrm{m}$ (red), and $6 \mu \mathrm{m}$ (blue). Data points were spaced by $0.5 \mu \mathrm{m}$ in the $x$ direction.

amplitudes of the normal stresses are significantly larger than the differences between normal stresses.

Figure 3 displays $\mathrm{DOP}_{100}$ for three different widths of the dielectric stripe. Measurements were made over an area removed from the edges of the InP sample and then averaged over the $y$ direction to reduce noise. The lengths of the $\mathrm{SiN}_{\mathrm{x}}$ stripes run along the $y$ direction, and by symmetry, $\mathrm{DOP}_{100}$ should have the same values for any value of $y$. See Fig. 1 .

Note in Fig. 3 that $\mathrm{DOP}_{100}$ is $\approx 0$ away from the stripe as one would expect, goes negative as the stripe is approached, and then goes strongly positive, and is nonzero under the stripe. The nonzero DOP under the film suggests strongly that the strain caused by the $\mathrm{SiN}_{\mathrm{x}}$ film is anisotropic. Since $\mathrm{DOP}_{100}$ under the film is higher for narrower stripes, it suggests that the anisotropy of the strain induced by the $\mathrm{SiN}_{\mathrm{x}}$ film is not constant.

Figure 4 shows plots of the shift in peak for CL spectroscopy scans across the $\mathrm{SiN}_{\mathrm{x}}$ stripes for stripes of three different widths. Note that the data obtained from the $\mathrm{DOP}_{100}$ and $\mathrm{CL}_{100}$ measurements show similar shapes and trends. Both the $\mathrm{DOP}_{100}$ and $\mathrm{CL}_{100}$ data are $\approx 0$ far outside the stripe, go negative as the stripe is approached, then strongly positive at the edge of the stripe and reduce. Near the midpoint of the width of the stripe, both $\mathrm{DOP}_{100}$ and $\mathrm{CL}_{100}$ decrease as the width of the stripe increases. The $\mathrm{DOP}_{100}$ scans tend to show more detail under the $\mathrm{SiN}_{\mathrm{x}}$ film than is observed in the peak shifts from the

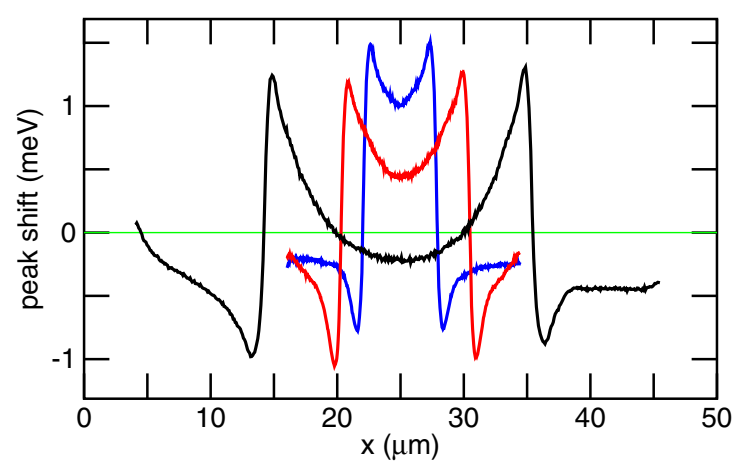

Fig. 4. Spectral shifts from the $C_{100}$ measurements across $S_{i N}$ stripes with widths of $20 \mu \mathrm{m}$ (black), $10 \mu \mathrm{m}$ (red), and $6 \mu \mathrm{m}$ (blue). Data points were spaced by $\lesssim 0.08 \mu \mathrm{m}$ in the $x$ direction.
$\mathrm{CL}_{100}$. It is not obvious from the $\mathrm{CL}_{100}$ peak-shift data that the in-plane strain in the film is not biaxial, i.e., $\epsilon_{x x} \neq \epsilon_{y y}$, whereas the non-zero value of $\mathrm{DOP}_{100}$ clearly indicates a non-biaxial strain.

The spatial resolution for the CL measurements is expected to be significantly better than that of the DOP measurements. However, the $\mathrm{CL}_{100}$ peak shifts under the $\mathrm{SiN}_{\mathrm{x}}$ stripe appear to be smoother than the $\mathrm{DOP}_{100}$ data under the stripe. The CL and DOP have different dependencies on the components of the strain tensor. Figures 3 and 4 demonstrate that different aspects of the strain tensor are obtained by the different measurement techniques.

As a first approximation, we use an analytic edge force model $[1-4,8]$ in a plane strain approximation to describe the stress in the $\operatorname{InP}$ owing to the $\mathrm{SiN}_{\mathrm{x}}$ stripe. Using this $2 \mathrm{D}$ approximation, we write the stress tensor as

$$
\left[\begin{array}{ccc}
\sigma_{x x}+\sigma_{x x}^{\prime} & 0 & \sigma_{x z} \\
0 & \sigma_{y y}^{\prime} & 0 \\
\sigma_{x z} & 0 & \sigma_{z z}
\end{array}\right],
$$

where $\sigma_{x x}, \sigma_{z z}$, and $\sigma_{x z}$ are components of stress from modeling the $\mathrm{SiN}_{\mathrm{x}}$ stripe with two edge forces [8] (pp. 97-100). DOP ${ }_{100}$ is non-zero in the center of the stripes, and this cannot be accounted for by the edge force model. Thus, we have included $\sigma_{x x}^{\prime}$ and $\sigma_{y y}^{\prime}$ and note that $\sigma_{x x}^{\prime} \neq \sigma_{y y}^{\prime}$.

A least squares fitting procedure was used to fit the stress tensor of Eq. (4) to the data shown in Figs. 3 and 4 through the dependence of $\mathrm{DOP}_{100}$ and $\mathrm{CL}_{100}$ peak shift on the components of the stress tensor. Parameters adjusted in the model included the stripe width $a$, the depths at which the $\mathrm{DOP}_{100}$ and $\mathrm{CL}_{100}$ signals originate, and the magnitudes of the line forces. The depth parameters are essential, as the presence of a discontinuity induces a singularity in the components of the stress tensor, which are obtained from the localized edge force. The amplitudes of the simulated peaks are directly governed by the depth at which the luminescence signals are generated.

Figure 5 shows least squares fits to the experimental data. It is clear that the analytic edge force model can be fit reasonably well to the $\mathrm{DOP}_{100}$ and $\mathrm{CL}_{100}$ data with a minimum number

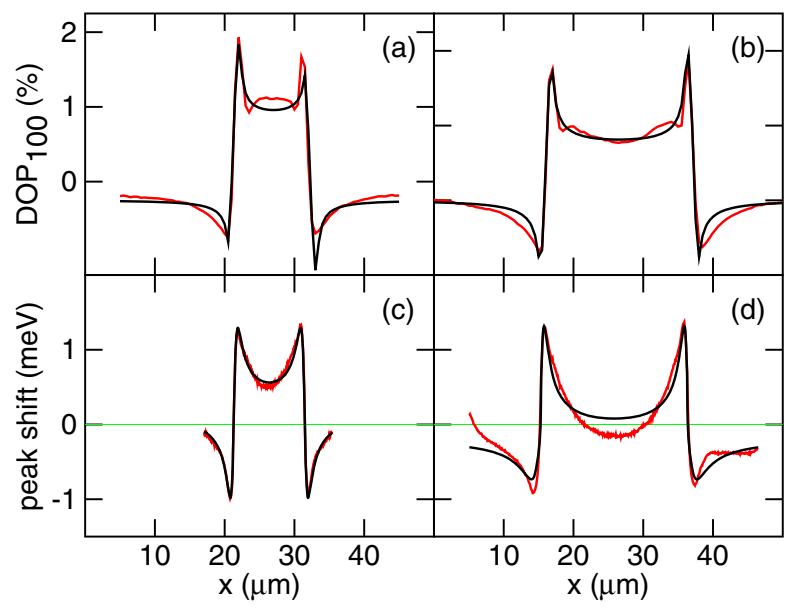

Fig. 5. Experimental (red) and simulated (black) $\mathrm{DOP}_{100}$ curves for the $\mathrm{SiN}_{\mathrm{x}}$ stripes with width $10 \mu \mathrm{m}$ (a) and $20 \mu \mathrm{m}$ (b); experimental and simulated $\mathrm{CL}_{100}$ curves for the $\mathrm{SiN}_{\mathrm{x}}$ with widths $10 \mu \mathrm{m}$ (c) and $20 \mu \mathrm{m}(\mathrm{d})$. 
DOP

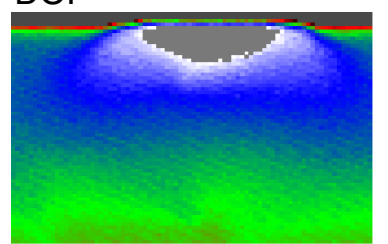

(a) measured

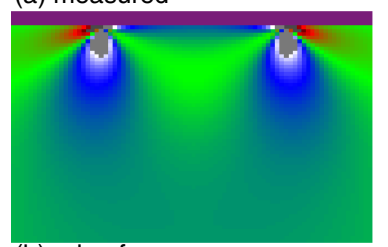

(b) edge force

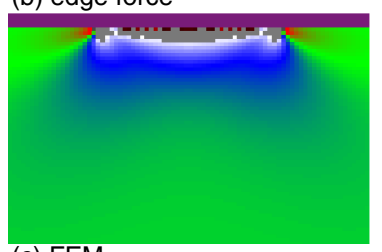

(c) FEM

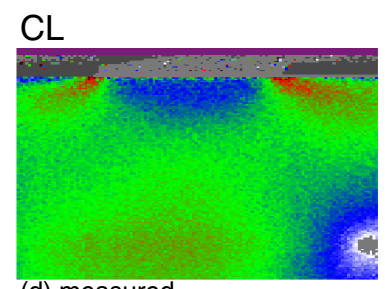

(d) measured

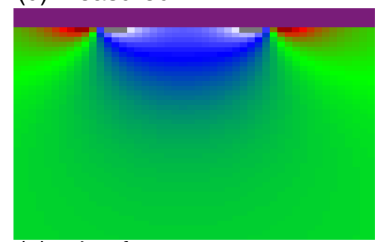

(e) edge force

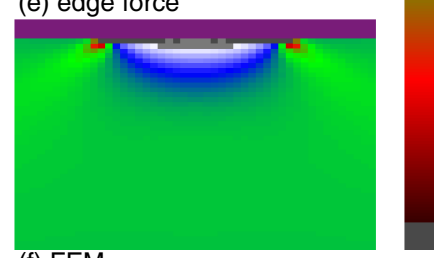

(f) FEM

Fig. 6. False color maps of $\mathrm{DOP}_{011}$ for $a=20 \mu \mathrm{m}$ stripes and $\mathrm{CL}_{011}$ maps for $a=6 \mu \mathrm{m}$ stripes. (a) and (d) are measured, (b) and (e) are edge force simulations, and (c) and (f) are FEM simulations. The color bar on the right-hand side shows the mapping of amplitude to color. The gray boxes on the ends of the bar map values that are out of range for the linear mappings between the two end points.

of fitting parameters. However, it is also clear that the simple edge force model does not predict the fine structure found for measurements under the $\mathrm{SiN}_{\mathrm{x}}$ stripes. It was found that the magnitudes of the positive peaks on either side of the stripe are sensitive to the fitting parameter used to represent the depth of the source of the luminescence.

Figure 6 shows measured and simulated $\mathrm{DOP}_{011}$ and $\mathrm{CL}_{011}$ for $20 \mu \mathrm{m}$ and $6 \mu \mathrm{m} \mathrm{SiN}$ stripes, respectively. In contrast to the simulations for measurements on a (100) surface, the FEM simulations for $\mathrm{DOP}_{011}$ provide better results than do the edge force simulations. This suggests that the deformation is more complex than application of a strained film of rectangular cross section (see Fig. 1) to a substrate. Thus, accurate measurements are required to inform simulations so that accurate information, such as photo-elastic changes of refractive index or strain distributions, can be obtained from simulations and used to design and optimize photonic devices. The 3D FEM simulations used zero stress boundary conditions at free surfaces and the orthotropic constants of Ref. [9] for InP.

In Fig. 6, any calculations with the analytic edge force model used parameters obtained from fits to the $\mathrm{DOP}_{100}$ and $\mathrm{CL}_{100}$ data. For the $\mathrm{DOP}_{011}$ maps shown in Fig. 6, the maps are $32 \mu \mathrm{m}$ high $\times 41 \mu \mathrm{m}$ wide, whereas for the $\mathrm{CL}_{011}$ maps, areas of $12 \mu \mathrm{m} \times 12 \mu \mathrm{m}$ are shown. The color bar on the right-hand

side in Fig. 6 indicates the sign and relative magnitude of the data. The mid-point of the color bar is zero. Negative values are mapped to colors below the mid-point of the color bar. For the DOP data, the colors at the top and bottom correspond to $\pm 1.46 \%$. For the CL data, the end points of the scale are $\pm 0.292 \mathrm{meV}$. The higher spatial resolution of the measured $\mathrm{CL}_{011}$ data, as compared to the measured $\mathrm{DOP}_{011}$ data, is apparent in Fig. 6.

In summary, we have used two different optical techniques to investigate the mechanical deformation of $\mathrm{InP}$ under $\mathrm{SiN}_{\mathrm{x}}$ films on (100) InP substrates. The two techniques, DOP of PL and peak-shift CL spectroscopy, provide complementary information on the deformation of the InP material under study. Consistency in results obtained with the two optical techniques gives confidence that the techniques are indeed measuring deformations of the material and not artifacts, and gives information on the components of the stress and strain tensors that could not be obtained with application of only one of the techniques, and provides feedback for the selection of accurate approaches to simulation.

Measurements on the samples were compared to simulations from a simple analytic edge force model and simple FEM simulations. The edge force model simulates reasonably well the experimental data for measurements from the (100) surface but does not simulate well measurements from the cleaved facet of the sample. The FEM simulates reasonably well the measurements from the cleaved facet. Here reasonably well means that the simulation captures the gross features of the data but misses some of the fine detail. This fine detail might be important in the simulation of photo-elastic changes in the refractive index or deformations that might be of interest in photonic device operation and reliability.

This work should help to motivate accurate measurements of the deformation of III-V materials, from which accurate simulations of deformations and changes in index and gain can be made. These results should help to "close the loop" for optimization of photo-elastic waveguides in particular, and photonic devices in general, by providing paths for accurate feedback.

\section{REFERENCES}

1. P. A. Kirkby, P. R. Selway, and L. D. Westbrook, J. Appl. Phys. 50, 4567 (1979).

2. S. M. Hu, J. Appl. Phys. 50, 4661 (1979).

3. I. De Wolf, H. E. Maes, and S. K. Jones, J. Appl. Phys. 79, 7148 (1996).

4. J. Yang and D. T. Cassidy, J. Appl. Phys. 77, 3382 (1995).

5. H. Rho, H. E. Jackson, and B. L. Weiss, J. Appl. Phys. 90, 276 (2001).

6. C.-L. Tien and T.-W. Lin, Appl. Opt. 51, 7229 (2012).

7. J.-M. Bonard, J.-D. Ganière, B. Akamatsu, D. Araújo, and F.-K. Reinhart, J. Appl. Phys. 79, 8693 (1996).

8. S. P. Timoshenko and J. N. Goodier, Theory of Elasticity, 3rd ed. (McGraw-Hill, 1970).

9. D. T. Cassidy, S. K. K. Lam, B. Lakshmi, and D. M. Bruce, Appl. Opt. 43, 1811 (2004).

10. G. D. Pitt, Solid State Commun. 8, 1119 (1970). 